\title{
Ridgelet-type Frame
}

\section{Decompositions for Sobolev}

\section{Spaces related to Linear Transport}

\section{Journal Article}

\section{Author(s):}

Grohs, Philipp

Publication date:

2012-04

\section{Permanent link:}

https://doi.org/10.3929/ethz-b-000338092

\section{Rights / license:}

In Copyright - Non-Commercial Use Permitted

\section{Originally published in:}

Journal of Fourier Analysis and Applications 18(2), https://doi.org/10.1007/s00041-011-9206-1 


\title{
Ridgelet-type Frame Decompositions for Sobolev Spaces related to Linear Transport
}

\author{
P. Grohs
}

Received: 22 December 2010 / Revised: 25 October 2011 / Published online: 24 November 2011

(C) Springer Science+Business Media, LLC 2011

\begin{abstract}
In this paper we study stability properties of ridgelet and curvelet frames for mixed-smoothness Sobolev spaces with norm $\|f\|_{s}=\|f\|_{L_{2}\left(\mathbb{R}^{d}\right)}+\| s$. $\nabla f \|_{L_{2}\left(\mathbb{R}^{d}\right)}$. Here $s \in \mathbb{S}^{d-1}$ is a transport direction and $\nabla$ denotes the gradient of $f$. Such spaces arise as domains of linear, first order transport equations. The main result of this paper is that ridgelet frames are stable in $\|\cdot\|_{s}$ regardless of $s$, while curvelet frames are not. To show the second statement we explicitly construct functions $f, g$ whose curvelet coefficients have all the same modulus but $\|f\|_{s}<\infty$ and $\|g\|_{s}=\infty$.
\end{abstract}

Keywords Sobolev spaces · Ridgelets $\cdot$ Curvelets $\cdot$ Transport equations

Mathematics Subject Classification (2000) $42 \mathrm{C} 40 \cdot 46 \mathrm{E} 30 \cdot 35 \mathrm{~F} 05$

\section{Introduction}

Motivated by the fact that classical isotropic representation systems like wavelets do not perform well for high dimensional functions with singularities along hypersurfaces, a whole arsenal of new representation systems for $L_{2}\left(\mathbb{R}^{d}\right)$ has enriched the field of harmonic analysis in the last decades, specifically for the case $d=2,3$. To give an incomplete picture of these developments we only mention ridgelets [2], curvelets [4], contourlets [9], bandlets [17], shearlets [14], wedgelets [11], ... The main goal of all these representation systems is to properly handle data with anisotropic features.

Communicated by Stephan Dahlke.

This work has been supported by the European Research Council under Grant ERC Project STAHDPDE No. 247277.

P. Grohs $(\bowtie)$

ETH Zürich, Seminar for Applied Mathematics, Rämistraße 101, 8092 Zürich, Switzerland

e-mail: pgrohs@math.ethz.ch 
A breakthrough in this direction has been obtained in [6] with the introduction of curvelets. There it is shown that one can construct a nonadaptive representation system that can approximate bivariate, piecewise $C^{2}$-functions away from $C^{2}$ curves with an (almost) optimal rate in terms of nonlinear approximation.

Shortly afterwards shearlets have been introduced in [14]. They share the desirable properties of curvelets with the additional advantage of a 'faithful digital transform', see e.g. [16] for details.

Curvelets have evolved from two ideas. The first one comes from the work of Hart Smith [20], who constructed curvelet-like systems to construct parametrices for hyperbolic PDEs. The second idea is based on a refinement of so-called ridgelets which are essentially ridge functions with oscillations across the ridge [2].

With all these novel constructions at hand it is natural to ask for which function spaces they are stable in the sense of a norm equivalence between the function space norm and a discrete norm on the sequence of transform coefficients. This question is especially important for solving operator equations, where Sobolev-type spaces are of particular significance, since they arise as domains of definition of various differential operators.

Not much is known in this direction for curvelets, shearlets or ridgelets besides the simple fact that they are stable in the classical Sobolev spaces (we are not aware of a published proof for this fact but it is a simple consequence of the frequency support properties of these systems). However, since already wavelets provide stable bases for these spaces this fact is not really useful.

In the present paper we investigate so-called Sobolev spaces of mixed smoothness, to be defined below, which arise as domains of definition for linear first order transport operators with constant transport direction. Operators of this kind are important in several applications such as radiative transport [19, 21].

It is well-known (and easy to see) that wavelets do not provide stable bases for such spaces. Here we show that ridgelets are stable while curvelets are not.

This result raises substantial hope that it is possible to construct stable solvers for first order transport equations with ridgelet discretizations in the spirit of [8] - a task that we presently investigate.

\subsection{Motivation}

The theory of wavelets shows us that in order to fully understand the properties of a representation system like curvelets or ridgelets, it is crucial to understand their approximation spaces. Only little is known in this direction for curvelets and ridgelets, we are only aware of the studies [1,2]. However, if one wants to use e.g. a curvelet system for the solution of a partial differential equation, one usually needs to investigate stability properties in terms of certain Sobolev norms. To give an example, we consider the simple transport equation

$$
s \cdot \nabla f+\kappa f=g,
$$

where $s \in \mathbb{S}^{d-1}$, the unit sphere in $\mathbb{R}^{d}, \nabla$ is the gradient of $f \in L_{2}\left(\mathbb{R}^{d}\right)$ and $\kappa$ is (say) a Lipschitz function, bounded from above and below. Given $g$ we would like to 
find $f$ satisfying certain boundary conditions. Of course this is a very simple equation but in order to solve it e.g. with a Ritz-Galerkin-type scheme, it is necessary to study the stability properties of our system of functions with respect to the norm $\|f\|_{s}^{2}:=\|f\|_{2}^{2}+\|s \cdot \nabla f\|_{2}^{2}$. We refer to norms of this type as mixed-smoothness Sobolev norm. A more interesting equation arises if $f \in L_{2}\left(\mathbb{R}^{d} \times \mathbb{S}^{d-1}\right)$ is also a function of $s$ and $s$ varies in $\mathbb{S}^{d-1}$. Equations of this type govern the radiation intensity in radiative transfer theory [19] and also arise in several other places. A stable and efficient solution method for such equations is currently not available. In order to arrive at a stable formulation, it is essential to have a representation system of functions that is simultaneously stable for $\|\cdot\|_{s}$ with respect to all angles $s \in \mathbb{S}^{d-1}$. In view of the directionality present in the norm it is clear that a stable representation cannot be isotropic. For instance, wavelet systems are not stable with respect to the norm $\|\cdot\|_{s}$ for all $s$, as can be easily seen. Due to their inherent directionality, natural candidates for stable systems are given by curvelet or ridgelet systems.

\subsection{Contributions}

Our first result, Theorem 10, is that ridgelets satisfy the desired stability property. The second main result, Theorem 18, of this paper is that curvelets are not stable with respect to $\|\cdot\|_{s}$, regardless of $s$. We show the latter by giving explicit counterexamples for $d=2$. In view of solving operator equations these results have two main implications:

- ridgelet-based methods are very promising candidates for developing RitzGalerkin-type solvers for transport problems, and

- curvelet-based methods cannot be used to solve transport problems, at least with conventional Ritz-Galerkin schemes.

Our results also remain valid for more general norms of the following type: Given a finite sequence $(\mathbf{s}, \alpha)=\left(s_{i}, \alpha_{i}\right)_{i=1}^{n}, \alpha \in \mathbb{R}_{+}, s_{i} \in \mathbb{S}^{d-1}$ we define

$$
\|f\|_{(\mathbf{s}, \alpha)}:=\|f\|_{2}+\sum_{i=1}^{n}\left\|\left(s_{i} \cdot \nabla\right)^{\alpha_{i}} f\right\|_{2},
$$

where $\left(s_{i} \cdot \nabla\right)^{\alpha_{i}}$ should be interpreted in the sense of pseudodifferential calculus [15], see Definition 11. Theorem 12 says that ridgelets are also stable w.r.t. these more general norms.

\subsection{Notation}

We fix a dimension $d \geq 2$ with $d \in \mathbb{N}$. For two vectors $u, v \in \mathbb{R}^{d}$ we denote their inner product by $u \cdot v$. For $f \in L_{1}\left(\mathbb{R}^{d}\right) \cap L_{2}\left(\mathbb{R}^{d}\right)$ we denote by $\hat{f}$ its Fourier transform $\hat{f}(\xi):=\left(\frac{1}{2 \pi}\right)^{d / 2} \int_{\mathbb{R}^{d}} f(x) \exp (i x \cdot \xi) d x$ and extend this operation to $L_{2}\left(\mathbb{R}^{d}\right)$. We shall frequently use the notation $A \lesssim B$ to indicate that the quantity $A$ is bounded by a constant times $B$. If $A \lesssim B$ and $B \lesssim A$ we shall also write $A \sim B$. For a function $f$ we denote by $\nabla f$ its gradient. We will also use the notation $\delta_{j j^{\prime}}$ for the Kronecker function which is one if $j=j^{\prime}$ and zero otherwise. The symbol $B_{\mathbb{S}^{d-1}}(s, r)$ shall 
denote the geodesic ball of radius $r$ in $\mathbb{S}^{d-1}$ around $s \in \mathbb{S}^{d-1}$. The symbol $|\cdot|$ will be used to denote the absolute value on $\mathbb{C}$, the Euclidean norm on $\mathbb{R}^{d}$ and the cardinality of a set.

\section{Ridgelet Tight Frames}

We start by constructing a tight frame of ridgelets for $L_{2}\left(\mathbb{R}^{d}\right)$. Recall that a system $\left(\psi_{\lambda}\right)_{\lambda \in \Lambda}$ of $L_{2}$ functions is called a tight frame of $L_{2}\left(\mathbb{R}^{d}\right)$ if

$$
\|f\|_{2}^{2}=\sum_{\lambda \in \Lambda}\left|\left\langle f, \psi_{\lambda}\right\rangle\right|^{2} \quad \text { for all } f \in L_{2}\left(\mathbb{R}^{d}\right) .
$$

If (1) only holds with $\sim$ instead of $=$, we speak of a frame. The main property of a frame is that any $f \in L_{2}\left(\mathbb{R}^{d}\right)$ can be stably decomposed into, and reconstructed from the sequence $\left(\left\langle f, \psi_{\lambda}\right\rangle\right)_{\lambda \in \Lambda}$ - with some possible redundancy in the decomposition [7].

\subsection{Preliminaries on Ridgelets}

Since there does not yet exist a uniform definition of ridgelets in the literature-the same is true for curvelets, by the way-we start by listing the various constructions that go by this name. As it is the case for essentially all transforms in harmonic analysis, the crucial property is the time-frequency localization of the functions and this is the viewpoint that we will adapt in the present paper. It can be shown that all the constructions described below are equivalent in terms of their approximation properties, see Remark 8.

In [2] a ridgelet transform has been introduced using a univariate (oscillatory) function $\psi$ by mapping a function $f \in L_{2}\left(\mathbb{R}^{d}\right)$ to its transform coefficients

$$
\left\langle f(x), a^{1 / 2} \psi(a s \cdot x-t)\right\rangle, \quad s \in \mathbb{S}^{d-1}, t \in \mathbb{R}, a \in \mathbb{R}_{+} .
$$

The function $x \mapsto a^{1 / 2} \psi(a s \cdot x-t)$ is a ridge function (hence the name ridgelet) which only varies in the direction $s$. In particular this function is not in $L_{2}\left(\mathbb{R}^{d}\right)$ and therefore, (2), as it stands, makes no sense. For continuously varying parameters $(a, s, t)$ one can still provide a stable reconstruction formula (similar to the continuous Fourier transform), but when we want to discretize the parameters $a, s, t$ e.g. to $a=2^{j}, j \in \mathbb{N}, t \in \mathbb{Z}$ and $s$ in some discrete and uniformly distributed subset of $\mathbb{S}^{d-1}$ with cardinality $\sim 2^{j}$ —we need to evaluate (2) pointwise which makes no sense for general $f \in L_{2}\left(\mathbb{R}^{d}\right)$. Nevertheless, Candes showed in [2] that for compactly supported functions $f \in L_{2}\left([0,1]^{d}\right)$ a stable reconstruction can be given from the transform coefficients sampled on a discrete set. In other words for $f \in L_{2}\left([0,1]^{d}\right)$ discrete ridgelet frames can be constructed.

By relaxing the definition of a ridgelet a little, it is possible to construct frames for $L_{2}\left(\mathbb{R}^{d}\right)$. The idea is that $\psi(a s \cdot x-t)$ can also be written as $a^{1 / 2} \rho\left(D_{a} R_{s} x-t\right)$, where $D_{a}=\operatorname{diag}(a, 1, \ldots, 1), R_{s}$ an orthogonal transform mapping $s \in \mathbb{S}^{d-1}$ to the vector $(1,0, \ldots, 0), t \in \mathbb{R}^{d}$ and

$$
\rho(x)=\psi\left(x_{1}\right) .
$$



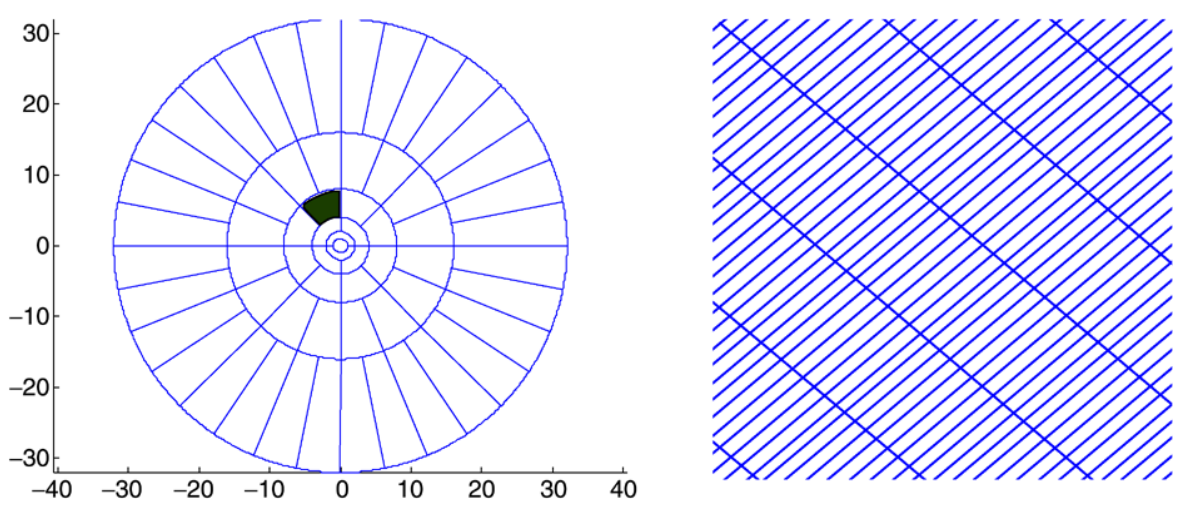

Fig. 1 Left: Frequency space decomposition as indicated by the ridgelet frame. The essential support of $\hat{\psi}_{3,3}$ is colored. Right: Translational grid of the frame elements $\rho_{3,3, k}, k \in \mathbb{Z}^{2}$. The aspect ratio of the tiles is $1 \sim 2^{-3}$

If we allow the function $\rho$ to vary also a little in the other coordinate directions besides $(1,0, \ldots, 0)$ so as to make $\rho \in L_{2}\left(\mathbb{R}^{d}\right)$, it can be shown that the parameters $a, s, t$ can be sampled discretely to yield a frame for $L_{2}\left(\mathbb{R}^{d}\right)$. In this spirit, one might define a ridgelet system as a system of functions which are of the form

$$
a^{1 / 2} \rho\left(D_{a} R_{s} x-t\right)
$$

with some $\rho \in L_{2}\left(\mathbb{R}^{d}\right)$, which is oscillatory in the first coordinate, and for the parameters $(a, s, t)$ ranging in some discrete set - typically $a=2^{j}, j \in \mathbb{N}, t \in \mathbb{Z}^{d}$ and $s$ in a uniformly distributed subset of $\mathbb{S}^{d-1}$ of cardinality $\sim 2^{j}$.

A yet more general viewpoint is to characterize ridgelets by their localization properties in space and frequency_-without enforcing the rigid condition of being a frame of functions exactly of the form (4). This is the viewpoint that we shall take in this paper. For us, a ridgelet system is a system of functions that is adapted to the partitioning of frequency space outlined in Fig. 1, left.

This partitioning consists of polar wedges with opening angle $\sim 2^{-j}$ and contained in the dyadic corona $2^{j} \leq|\xi|<2^{j+1}$. An easy computation that takes into account the oscillatory behavior of $\rho$ in the first coordinate, reveals that indeed the functions as defined in (4) have approximate frequency support in these wedges.

This viewpoint, which goes by the name of decomposition spaces [13], has been taken before for wavelet, Gabor, curvelet or shearlet systems.

The main advantage of this approach is that it allows for particularly simple tight frame constructions. On the other hand, the desirable approximation properties of the original definitions still remain valid in the more general context based on decomposition spaces.

It should be noted that yet another definition of ridgelets is given in [10], see also [12] for more information about the various different constructions of curvelets and ridgelets. 


\subsection{A New Construction}

In this section we present a novel construction of a ridgelet tight frame. Since a ridgelet system is a system of functions which is adapted to the frequency tiling outlined in Fig. 1 into angular wedges of opening angle $\sim 2^{-j}$ and height $\sim 2^{j}, j \in \mathbb{N}$, the first step in our construction is to find a partition-of-unity which is adapted to this tiling.

Due to the fact that we are interested in discrete systems we first need to find discrete sampling points on the sphere. The following lemma shows that one can always find reasonable uniformly distributed points on the sphere with a prescribed distance. A proof can be found e.g. in [1, Lemma 7].

Lemma 1 Let $\mathbb{S}^{d-1}$ be the unit sphere equipped with the geodesic metric inherited from the Euclidean ambient space $\mathbb{R}^{d}$. Then for any $r>0$ there exist $L \sim r^{-1}$, points $\left(s_{l}\right)_{l=1}^{L}$ on $\mathbb{S}^{d-1}$ and a constant $A$ (independent of $r$ ) such that

$$
\bigcup_{l=1}^{L} B_{\mathbb{S}^{d-1}}\left(s_{l}, r\right)=\mathbb{S}^{d-1},
$$

and

$$
\max _{l=1}^{L}\left|\left\{l^{\prime} \neq l: B_{\mathbb{S}^{d-1}}\left(s_{l}, 2 r\right) \cap B_{\mathbb{S}^{d-1}}\left(s_{l^{\prime}}, 2 r\right) \neq \emptyset\right\}\right| \leq A .
$$

In what follows we will construct a partition-of-unity for the ridgelet frequency tiling. To this end we will use radial coordinates and build our partition functions from a window function $W$ for the radial variable and window functions $V^{(j)}$ for the angular variable corresponding to scale $j$. We now give the detailed definition.

We denote by $e_{1}$ the unit vector $(1,0, \ldots, 0) \in \mathbb{S}^{d-1}$.

Definition 2 We fix smooth, nonnegative functions $V^{(j)}: \mathbb{S}^{d-1} \rightarrow \mathbb{R}, j \in \mathbb{N}, W$ : $\mathbb{R}_{+} \rightarrow \mathbb{R}$ and $W^{(0)}: \mathbb{R}_{+} \rightarrow \mathbb{R}$ with the following properties:

(i) $\operatorname{supp} V^{(j)} \subset B_{\mathbb{S}^{d-1}}\left(e_{1}, 2 \cdot 2^{-j}\right)$,

(ii) $V^{(j)}(s) \geq 1$ for all $s \in B_{\mathbb{S}^{d-1}}\left(e_{1}, 2^{-j}\right)$,

(iii) $V^{(j)}(s) \leq 2$ for all $s \in B_{\mathbb{S}^{d-1}}\left(e_{1}, 2 \cdot 2^{-j}\right)$,

(iv) $\operatorname{supp} W \subset(1 / 2,2)$,

(v) $W(r) \geq 1$ for all $r \in(3 / 4,3 / 2)$,

(vi) supp $W^{(0)} \subset[0,2)$,

(vii) $W^{(0)}(r) \geq 1$ for all $r \leq 1$.

We define $R_{s}$ as an orthogonal transform which maps the point $s \in \mathbb{S}^{d-1}$ to $e_{1}$. We also pick a sequence of sampling points $\left(s_{j, l}\right)_{l=1}^{L_{j}}$ satisfying the conditions of Lemma 1 with $r=2^{-j}, j \geq 1$. 
Lemma 3 There exist positive constants $C_{1}, C_{2}$ so that

$$
C_{1}<\Phi(\xi):=\sum_{j, l} W\left(2^{-j}|\xi|\right)^{2} V^{(j)}\left(R_{s_{j, l}} \frac{\xi}{|\xi|}\right)^{2}+W^{(0)}(|\xi|)^{2}<C_{2} \quad \text { for all } \xi \in \mathbb{R}^{d}
$$

Proof We first show the existence of the constant $C_{1}$ : since by (5), for any $\xi \in \mathbb{R}^{d}$ there exists $j, l$ such that $\xi$ has the representation $\left(|\xi|, \frac{\xi}{|\xi|}\right)$ with $|\xi| \in 2^{j}[3 / 4,3 / 2]$ and $\frac{\xi}{|\xi|} \in B_{\mathbb{S}^{d-1}}\left(s_{j, l}, 2^{-j}\right)$. From properties (ii), (v) it follows that

$$
\Phi(\xi) \geq W\left(2^{-j}|\xi|\right)^{2} V^{(j)}\left(\frac{\xi}{|\xi|}\right)^{2} \geq 1
$$

which gives the lower bound. The upper bound $C_{2}$ follows by noting that by (iii) and (6) any $\xi \in \mathbb{R}^{d}$ lies at most in the support of finitely many summands with bounded magnitude.

The previous definitions enable us to finally define the frequency windows which are adapted to the ridgelet tiling.

Definition 4 We define the following functions in terms of their Fourier transforms:

$$
\hat{\psi}_{j, l}(\xi):=\frac{W\left(2^{-j}|\xi|\right) V^{(j)}\left(R_{s_{j, l}} \frac{\xi}{|\xi|}\right)}{\sqrt{\Phi(\xi)}}, \quad j \geq 1, l=1, \ldots, L_{j}
$$

and

$$
\hat{\psi}_{0}(\xi):=\frac{W(|\xi|)}{\sqrt{\Phi(\xi)}} .
$$

Observe that by (7) the division by $\Phi$ is well-defined and the functions $\psi_{j, l}, \psi_{0}$ are in $L_{2}\left(\mathbb{R}^{d}\right)$.

Definition 5 We define the wedges

$$
P_{0}:=\{\xi:|\xi| \leq 2\}, \quad P_{j, l}:=\left\{\xi: 2^{j-1}<|\xi| \leq 2^{j+1}, \frac{\xi}{|\xi|} \in B_{\mathbb{S}^{d-1}}\left(s_{j, l}, 2^{-j} 2\right)\right\}
$$

It follows that

$$
\text { supp } \hat{\psi}_{0} \subset P_{0} \quad \text { and } \quad \operatorname{supp} \hat{\psi}_{j, l} \subset P_{j, l} .
$$

We can now derive a semidiscrete representation formula for $L_{2}\left(\mathbb{R}^{d}\right)$.

Proposition 6 We have

$$
\|f\|_{2}^{2}=\left\|f * \psi_{0}\right\|_{2}^{2}+\sum_{j, l}\left\|f * \psi_{j, l}\right\|_{2}^{2} .
$$


Proof The proof follows standard arguments, therefore we will ignore technical details like convergence issues. From the definition of $\psi_{0}, \psi_{j, l}$ and $\Phi$ it follows that

$$
\hat{\psi}_{0}^{2}(\xi)+\sum_{j, l} \hat{\psi}_{j, l}(\xi)^{2}=1
$$

Therefore we have

$$
\begin{aligned}
\|f\|_{2}^{2} & =\int_{\mathbb{R}^{d}}|\hat{f}(\xi)|^{2} d \xi \\
& =\int_{\mathbb{R}^{d}} \sum_{j, l} \hat{\psi}_{j, l}(\xi)^{2}|\hat{f}(\xi)|^{2} d \xi+\int_{\mathbb{R}^{d}} \psi_{0}(\xi)^{2}|\hat{f}(\xi)|^{2} d \xi \\
& =\left\|f * \psi_{0}\right\|_{2}^{2}+\sum_{j, l}\left\|f * \psi_{j, l}\right\|_{2}^{2} .
\end{aligned}
$$

We go on to construct a tight frame decomposition of $L_{2}\left(\mathbb{R}^{d}\right)$ by discretizing the translational parameter in the convolutions in (8). To this end define the functions

$$
\begin{aligned}
& \rho_{j, l, k}(x):=2^{-j / 2} T_{x_{j, l, k}} \psi_{j, k}, \quad \varphi_{k}:=T_{k} \psi_{0}, \\
& k=\left(k_{1}, \ldots, k_{d}\right) \in \mathbb{Z}^{d}, \quad x_{j, l, k}:=R_{s_{j, l}}^{*}\left(2^{-j} k_{1}, k_{2}, \ldots, k_{d}\right)^{T}, \\
& \quad \text { and } \quad T_{y} f(\cdot):=f(\cdot-y) .
\end{aligned}
$$

Theorem 7 The system

$$
\left(\varphi_{k}\right)_{k \in \mathbb{Z}^{d}} \cup\left(\rho_{j, l, k}\right)_{j \geq 1, l \in\left[0, L_{j}\right], k \in \mathbb{Z}^{d}}
$$

constitutes a tight frame for $L_{2}\left(\mathbb{R}^{d}\right)$.

Proof In view of (8) we need to show that

$$
\left\|f * \psi_{j, l}\right\|_{2}^{2}=\sum_{k \in \mathbb{Z}^{d}}\left|\left\langle f, \rho_{j, l, k}\right\rangle\right|^{2}
$$

and

$$
\left\|f * \psi_{0}\right\|_{2}^{2}=\sum_{k \in \mathbb{Z}^{d}}\left|\left\langle f, \varphi_{k}\right\rangle\right|^{2}
$$

Since

$$
\left\|f * \psi_{j, l}\right\|_{2}^{2}=\int_{\mathbb{R}^{2}}|\hat{f}(\xi)|\left|\hat{\psi}_{j, l}(\xi)\right|^{2} d \xi=\int_{P_{j, l}}|\hat{f}(\xi)|\left|\hat{\psi}_{j, l}(\xi)\right|^{2} d \xi
$$

equation (9) is shown by noting that the system $\left(2^{-j / 2} \exp \left(i x_{j, l, k} \cdot \xi\right)\right)_{k \in \mathbb{Z}^{d}}$ constitutes an ONB of $L_{2}\left(P_{j, l}\right)$ (compare also Lemma 4.2 in [6]). Equation (10) is proven in the same way. 
Remark 8 Perhaps the best way to think of the functions $\rho_{j, l, k}$ is to write them as

$$
\rho_{j, l, k}(\cdot):=2^{j / 2} m^{(j, l, k)}\left(D_{j} R_{s_{j, l}} \cdot-k\right),
$$

where $D_{j}=\operatorname{diag}\left(2^{j}, 1, \ldots, 1\right)$. This way, the resemblance to (4) is seen. In our construction the functions $m^{(j, l, k)}$ are different for different indices but it is not difficult to show that this difference is only minor. To make this precise one would have to introduce the concept of ridgelet molecules as has been done in [3] for curvelets and in [18] for wavelets (the latter construction goes by the name 'Vaguelettes'). Then, adapting the proofs of [3, Sect. 2.4] one can show that any two systems of such molecules are almost orthogonal, implying the equivalence of their approximation properties. In Fig. 1 the localization properties of the ridgelet elements in space and frequency are depicted for the case $d=2$.

Remark 9 In applications, where data is given as a discrete function defined on a digital grid, it is very inconvenient to work with the operation of rotation. For curvelets the same problem arises and a solution has been proposed with the introduction of shearlets [16]. The main idea is to replace the rotation operations by appropriate shear operations, the latter being also defined on digital data. These same adaptions can also be carried out for ridgelets.

\section{Stability Properties}

\subsection{Main Result}

This section contains our first main result, namely the stability of the ridgelet tight frame with respect to the norm $\|\cdot\|_{s}$ as defined in the introduction. Our main stability theorem is as follows:

Theorem 10 Let $s \in \mathbb{S}^{d-1}$. Then we have the norm equivalence

$$
\|f\|_{2}^{2}+\|s \cdot \nabla f\|_{2}^{2} \sim \sum_{k \in \mathbb{Z}^{d}}\left|\left\langle f, \varphi_{k}\right\rangle\right|^{2}+\sum_{j, l}\left(1+2^{2 j}\left|s \cdot s_{j, l}\right|^{2}\right) \sum_{k \in \mathbb{Z}^{d}}\left|\left\langle f, \rho_{j, l, k}\right\rangle\right|^{2} .
$$

Proof We have

$$
\begin{aligned}
\|f\|_{2}^{2}+\|s \cdot \nabla f\|_{2}^{2} & \sim \int_{\mathbb{R}^{d}}\left(1+(s \cdot \xi)^{2}\right)|\hat{f}(\xi)|^{2} d \xi \\
= & \int_{\mathbb{R}^{2}}\left(1+(s \cdot \xi)^{2}\right)|\hat{f}(\xi)|^{2}\left|\hat{\psi}_{0}(\xi)\right|^{2} d \xi \\
& +\sum_{j, l} \int_{\mathbb{R}^{d}}\left(1+(s \cdot \xi)^{2}\right)|\hat{f}(\xi)|^{2}\left|\hat{\psi}_{j, l}(\xi)\right|^{2} d \xi
\end{aligned}
$$




$$
\begin{aligned}
= & \int_{P_{0}}\left(1+(s \cdot \xi)^{2}\right)|\hat{f}(\xi)|^{2}\left|\hat{\psi}_{0}(\xi)\right|^{2} d \xi \\
& +\sum_{j, l} \int_{P_{j, l}}\left(1+(s \cdot \xi)^{2}\right)|\hat{f}(\xi)|^{2}\left|\hat{\psi}_{j, l}(\xi)\right|^{2} d \xi
\end{aligned}
$$

The proof is complete if we can show that

(i)

$$
1+(s \cdot \xi)^{2} \sim 1, \quad \xi \in P_{0},
$$

and

(ii)

$$
1+(s \cdot \xi)^{2} \sim 1+2^{2 j}\left|s \cdot s_{j, l}\right|^{2}, \quad \xi \in P_{j, l}, j \geq 1 .
$$

Equivalence (i) is a simple consequence of the Cauchy-Schwartz inequality. We now prove (ii). Let us split the set of indices $j, l$ into

$$
\mathcal{I}_{0}:=\left\{(j, l):\left|s \cdot s_{j, l}\right| \leq 2^{-j} 20\right\}
$$

and

$$
\mathcal{I}_{1}:=\left\{(j, l):\left|s \cdot s_{j, l}\right|>2^{-j} 20\right\} .
$$

Note that we always have

$$
\left|s-s^{\prime}\right|<\sigma\left(s, s^{\prime}\right),
$$

$\sigma$ denoting the geodesic metric in $\mathbb{S}^{d-1}$.

- We start with $(j, l) \in \mathcal{I}_{0}$. By our assumptions on $(j, l)$ we then have

$$
1+2^{2 j}\left|s \cdot s_{j, l}\right|^{2} \sim 1
$$

On the other hand for any $\xi \in \mathbb{R}^{d}$ with representation $(|\xi|, s \xi)$, (13) together with the fact that

$$
s_{\xi} \in B_{\mathbb{S}^{d-1}}\left(s_{j, l}, 2^{-j} 2\right)
$$

and the Cauchy-Schwartz inequality imply that

$$
\left|s \cdot s_{j, l}-s \cdot s_{\xi}\right| \lesssim 2^{-j}
$$

and therefore

$$
|s \cdot \xi| \sim 2^{j}|s \cdot s \xi| \leq 2^{j}\left(\left|s \cdot s_{j, l}\right|+\left|s \cdot\left(s_{\xi}-s_{j, l}\right)\right|\right) \lesssim 1 .
$$

This implies that for $\xi \in P_{j, l},(j, l) \in \mathcal{I}_{0}$ we have

$$
1+(s \cdot \xi)^{2} \sim 1+2^{2 j}\left(s \cdot s_{j, l}\right)^{2} \sim 1
$$

and that is (i). 
- Now we let $(j, l) \in \mathcal{I}_{1}$ and $\xi \in P_{j, l}$ with spherical coordinates $\left(r, s_{\xi}\right)=\left(|\xi|, \frac{\xi}{|\xi|}\right)$, where $2^{j-1}<r<2^{j+1}$ and $\left|s \xi-s_{j, l}\right| \leq 2^{-j} 2$. Consider

$$
|s \cdot \xi|=r|s \cdot s \xi| \sim 2^{j}|s \cdot s \xi| .
$$

We need to show that

$$
\left|s \cdot s_{\xi}\right| \sim\left|s \cdot s_{j, l}\right| \text {. }
$$

We have (note that the division by $s \cdot s_{j, l}$ is permitted since $s \cdot s_{j, l} \neq 0$ by the assumption that $\left.(j, l) \in \mathcal{I}_{1}\right)$

$$
|s \cdot s \xi|=\left|s \cdot s_{j, l}+s \cdot\left(s_{\xi}-s_{j, l}\right)\right|=\left|s \cdot s_{j, l}\right|\left|1+\frac{s \cdot\left(s_{\xi}-s_{j, l}\right)}{s \cdot s_{j, l}}\right|
$$

and therefore it remains to bound the quantity

$$
\left|1+\frac{s \cdot\left(s_{\xi}-s_{j, l}\right)}{s \cdot s_{j, l}}\right|
$$

from above and below. We start with the estimate from below:

$$
\begin{aligned}
\left|1+\frac{s \cdot\left(s_{\xi}-s_{j, l}\right)}{s \cdot s_{j, l}}\right| & \geq 1-\left|\frac{s \cdot\left(s_{\xi}-s_{j, l}\right)}{s \cdot s_{j, l}}\right| \\
& \geq 1-\frac{2^{-j} 2}{2^{-j} 20} \geq 9 / 10 .
\end{aligned}
$$

On the other hand we have

$$
\begin{aligned}
\left|1+\frac{s \cdot\left(s_{\xi}-s_{j, l}\right)}{s \cdot s_{j, l}}\right| & \leq 1+\left|\frac{s \cdot\left(s_{\xi}-s_{j, l}\right)}{s \cdot s_{j, l}}\right| \\
& \leq 11 / 10 .
\end{aligned}
$$

This shows (15) and therefore we have

$$
1+(s \cdot \xi)^{2} \sim 1+2^{2 j}\left(s \cdot s_{j, l}\right)^{2},
$$

which is (ii).

In view of (12), (9) and (10) we have

$$
\begin{aligned}
& \|f\|_{2}^{2}+\|s \cdot \nabla f\|_{2}^{2} \\
& \sim \int_{P_{0}}\left(1+(s \cdot \xi)^{2}\right)|\hat{f}(\xi)|^{2}\left|\hat{\psi}_{0}(\xi)\right|^{2} d \xi \\
& \quad+\sum_{j, l} \int_{P_{j, l}}\left(1+(s \cdot \xi)^{2}\right)|\hat{f}(\xi)|^{2}\left|\hat{\psi}_{j, l}(\xi)\right|^{2} d \xi \\
& \sim\left\|f * \psi_{0}\right\|_{2}^{2}+\sum_{j, l}\left(1+2^{2 j}\left(s \cdot s_{j, l}\right)^{2}\right)\left\|f * \psi_{j, l}\right\|_{2}^{2}
\end{aligned}
$$




$$
=\sum_{k}\left|\left\langle f, \varphi_{k}\right\rangle\right|^{2}+\sum_{j, l, k}\left(1+2^{2 j}\left(s \cdot s_{j, l}\right)^{2}\right)\left|\left\langle f, \rho_{j, l, k}\right\rangle\right|^{2} .
$$

This proves the theorem.

\subsection{More General Spaces}

It is perhaps worth noting that ridgelet systems provide stable decompositions for a whole scale of spaces which we call mixed-smoothness Sobolev spaces. The definition is as follows:

Definition 11 For $\alpha>0$ we define the operator $(s \cdot \nabla)^{\alpha}$ acting on tempered distributions via

$$
\hat{f} \mapsto(s \cdot \xi)^{\alpha} \hat{f}
$$

For a finite sequence $(\mathbf{s}, \alpha):=\left(s_{i}, \alpha_{i}\right)_{i=1}^{n} \in\left(\mathbb{S}^{d-1} \times \mathbb{R}_{+}\right)^{n}$ we define the norm

$$
\|f\|_{(\mathbf{s}, \alpha)}:=\|f\|_{2}+\sum_{i=1}^{n}\left\|\left(s_{i} \cdot \nabla\right)^{\alpha_{i}} f\right\|_{2} .
$$

We have the following generalization of Theorem 10:

Theorem 12 Given $(\mathbf{s}, \alpha) \in\left(\mathbb{S}^{d-1} \times \mathbb{R}_{+}\right)^{n}$ we have the norm equivalence

$$
\|f\|_{(\mathbf{s}, \alpha)}^{2} \sim \sum_{k}\left|\left\langle f, \varphi_{k}\right\rangle\right|^{2}+\sum_{j, l, k}\left(1+\sum_{i=1}^{n} 2^{2 \alpha_{i} j}\left(s_{i} \cdot s_{j, l}\right)^{2 \alpha_{i}}\right)\left|\left\langle f, \rho_{j, l, k}\right\rangle\right|^{2} .
$$

The implicit constant depends on $n$.

Proof The proof is virtually identical to the proof of Theorem 10 and therefore we omit it.

\section{Instability of Curvelets}

In this section we prove the second main result, namely that curvelets are not stable with respect to $\|\cdot\|_{s}$, regardless of $s \in \mathbb{S}^{d-1}$. We construct our specific counterexample for $d=2$ but a modification along the same lines to arbitrary $d$ is possible, albeit with more notational overload.

In order to show the instability result, we construct two functions $f$ and $g$ in $L\left(\mathbb{R}^{2}\right)$ whose coefficients all have the same magnitude in a curvelet tight frame but $\|f\|_{s}<\infty$ and $\|g\|_{s}=\infty$. The idea for this construction is depicted in Fig. 3 .

In what follows we shall identify $\mathbb{S}^{1}$ with the interval $[-\pi, \pi)$.

First we give a construction of a curvelet tight frame convenient for our purposes. Similar to above we start with two window functions. 
Definition 13 We define univariate, nonnegative $C^{\infty}$ functions $W_{c}(r), V_{c}(t)$ so that

(i) $\operatorname{supp} W_{c} \subset\left(\frac{3}{4}, 4\right)$,

(ii) $\operatorname{supp} V_{c} \subset\left(-\frac{3}{4}, \frac{3}{4}\right)$,

(iii) $\sum_{j \in \mathbb{Z}} W_{c}^{2}\left(4^{-j} r\right)=1$, for all $r \in \mathbb{R}$ and

(iv) $\sum_{l \in \mathbb{Z}} V_{c}^{2}(t-l)=1$ for all $t \in \mathbb{R}$.

The construction of such window functions is standard in wavelet theory. For the convenience of the reader we sketch the construction of $W_{c}$ : Start with any smooth nonnegative function $\widetilde{W}$ which is supported in $\left[\frac{3}{4}, 4\right]$ and strictly positive on $\left(\frac{3}{4}, 4\right)$. Then define

$$
W_{c}(r):=\frac{\widetilde{W}(r)}{\left(\sum_{l \in \mathbb{Z}} \widetilde{W}^{2}\left(4^{-l} r\right)\right)^{1 / 2}} .
$$

This function satisfies (i). It also satisfies (iii), since

$$
\sum_{j \in \mathbb{Z}} W_{c}^{2}\left(4^{-j} r\right)=\sum_{j \in \mathbb{Z}} \frac{\widetilde{W}^{2}\left(4^{-j} r\right)}{\sum_{l \in \mathbb{Z}} \widetilde{W}^{2}\left(4^{-l} 4^{-j} r\right)}=\frac{\sum_{j \in \mathbb{Z}} \widetilde{W}^{2}\left(4^{-j} r\right)}{\sum_{l \in \mathbb{Z}} \widetilde{W}^{2}\left(4^{-l} r\right)}=1 .
$$

The construction of $V_{c}$ is similar. Observe that in view of (i) and (iii) resp. (ii) and (iv) we have

$$
W_{c}(r)=1 \quad \text { for } r \in(1,3) \quad \text { and } \quad V_{c}(t)=1 \quad \text { for } t \in\left(-\frac{1}{4}, \frac{1}{4}\right)
$$

Now, similar to the ridgelet definitions above we define

$$
s_{j, l}:=2 \pi l 2^{-j}, \quad L_{j}:=2^{j}-1
$$

and make the following definition:

Definition 14 We write $r, \omega$ for the polar variables of the frequency plane and define functions

$$
\hat{\varphi}^{c}(r, \omega)^{2}:=\sum_{j=0}^{-\infty} \sum_{l=0}^{L_{j}} W_{c}\left(4^{-j} r\right)^{2} V_{c}\left(\frac{2^{j}}{2 \pi}\left(\omega-s_{j, l}\right)\right)^{2}
$$

and

$$
\hat{\psi}_{j, l}^{c}(r, \omega):=W_{c}\left(4^{-j} r\right) V_{c}\left(\frac{2^{j}}{2 \pi}\left(\omega-s_{j, l}\right)\right)
$$

where $j>0$.

Sampling the translational variable on the integer grid yields the following definition. 

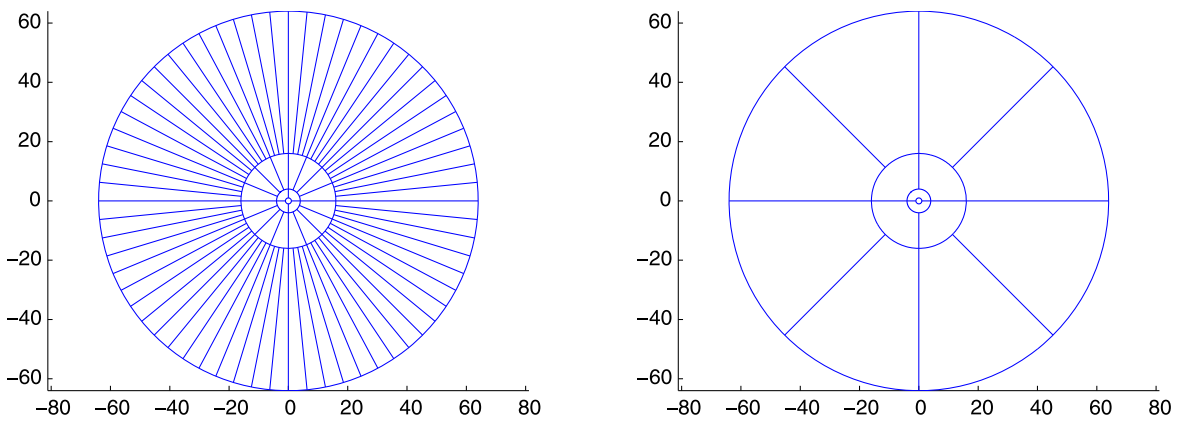

Fig. 2 Left: Illustration of the frequency partitioning related to ridgelets. Right: Illustration of the frequency partitioning related to curvelets

Definition 15 We define for $k=\left(k_{1}, k_{2}\right) \in \mathbb{Z}^{2}$ the functions

$$
\begin{gathered}
\varphi_{k}^{c}(\cdot):=\varphi^{c}(\cdot-k), \\
\gamma_{j, 0, k}(\cdot):=2^{-3 j / 2} \psi_{j, l}^{c}\left(\cdot-\left(4^{-j} k_{1}, 2^{-j} k_{2}\right)\right),
\end{gathered}
$$

and

$$
\gamma_{j, l, k}(\cdot):=\gamma_{j, 0, k}\left(R_{s_{j, l}} \cdot\right)
$$

The same arguments as above for the ridgelet case yield

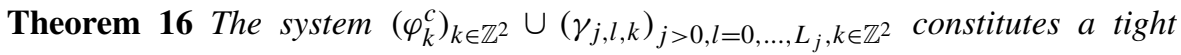
frame for $L_{2}\left(\mathbb{R}^{2}\right)$.

Definition 17 We call this tight frame a curvelet frame.

Observe that, unlike ridgelets, curvelets are supported in frequency wedges of aspect ratio $\sim 4^{j} \times 2^{j}$. This property is called parabolic scaling in the literature. The parabolic scaling allows curvelets to be well-localized in space and therefore better suited for applications where it is important to approximate curved singularities [5]. However, as we shall see, the increase in angular uncertainty of curvelets compared to ridgelets causes instability of the curvelet frame in mixed-smoothness Sobolev spaces, see Fig. 2 for an illustration. Indeed we will show the following:

Theorem 18 There exist two functions $f, g \in L_{2}\left(\mathbb{R}^{2}\right)$ such that

$$
\left\|e_{1} \cdot \nabla f\right\|_{2}<\infty
$$

(ii)

$$
\left\|e_{1} \cdot \nabla g\right\|_{2}=\infty, \text { and }
$$



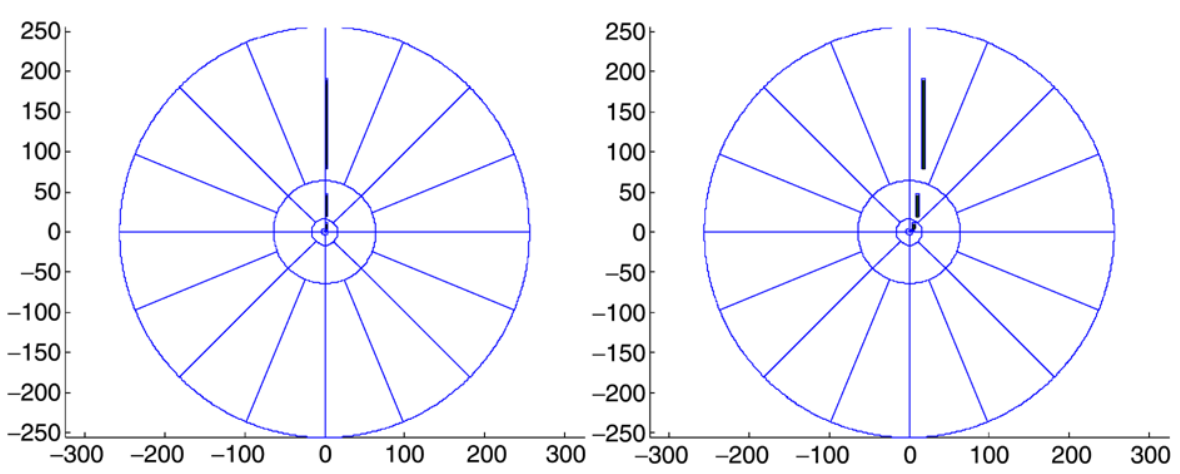

Fig. 3 Left: Illustration of the frequency support of the function $f$ which satisfies $\left\|e_{1} \cdot \nabla f\right\|_{2}<\infty$. Right: Illustration of the frequency support of the function $g$ with $\left\|e_{1} \cdot \nabla g\right\|_{2}=\infty$. The curvelet decomposition of the frequency plane cannot distinguish between these two functions. Note that in this figure, as opposed to Fig. 1, the aspect ratio of the angular wedges is $4^{j} \sim 2^{j}$

(iii)

$$
\left|\left\langle f, \gamma_{j, l, k}\right\rangle\right|=\left|\left\langle g, \gamma_{j, l, k}\right\rangle\right| \text { and }\left|\left\langle f, \varphi_{k}^{c}\right\rangle\right|=\left|\left\langle g, \varphi_{k}^{c}\right\rangle\right| \quad \text { for all indices } j, l, k \text {. }
$$

Proof First we define the rectangles

$$
Q_{j}:=\left[-\frac{1}{2} 2^{j}, \frac{1}{2} 2^{j}\right] \times\left[4^{j}, 24^{j}\right] .
$$

We have the following property for $j>2$ :

$$
\hat{\psi}_{j, l}^{c} \chi_{Q_{j}^{\prime}}=\delta_{j j^{\prime}} \delta_{l 2^{j-2}} .
$$

Indeed this follows immediately from the definition of $\hat{\psi}_{j, l}^{c}$. Here, $\chi_{Q_{j^{\prime}}}$ denotes the characteristic function of $Q_{j^{\prime}}$. Intuitively, equation (18) simply means that $Q_{j^{\prime}}$ only intersects with the support of $\hat{\psi}_{j^{\prime}, 2^{j^{\prime}-2}}^{c}$. Therefore, by the partition of unity property of the functions $\hat{\varphi}^{c}, \hat{\psi}_{j, l}^{c}$ it follows that the function $\hat{\psi}_{j^{\prime}, 2 j^{\prime}-2}^{c}$ restricted to $Q_{j^{\prime}}$ must equal 1. Also observe that $s_{j^{\prime}, 2 j^{\prime}-2}=\pi / 2$. Now we are ready to define the functions $f$ and $g$. Let $0<\varepsilon<4$.

$$
\hat{f}:=\sum_{j>2} 2^{-j-\varepsilon j} \chi_{[0,1] \times\left[4^{j}, 24^{j}\right]}
$$

and

$$
\hat{g}:=\sum_{j>2} 2^{-j-\varepsilon j} \chi_{\left[\frac{1}{2} 2^{j}-1, \frac{1}{2} 2^{j}\right] \times\left[4^{j}, 24^{j}\right]} .
$$

It follows that

$$
\|f\|_{2}^{2}=\sum_{j>2} 4^{-j-\varepsilon j} 4^{j}<\infty
$$


and

$$
\left\|e_{1} \cdot \nabla f\right\|_{2}^{2} \leq \sum_{j>2} 4^{-j-\varepsilon j} 4^{j}<\infty
$$

On the other hand,

$$
\|g\|_{2}^{2}=\sum_{j>2} 4^{-j-\varepsilon j} 4^{j}<\infty
$$

but

$$
\left\|e_{1} \cdot \nabla g\right\|_{2}^{2} \geq \sum_{j>2} 4^{-j-\varepsilon j} \frac{1}{4} 4^{j} 4^{j}=\infty .
$$

Now, let us inspect the curvelet coefficients of $f$. Observe that

$$
[0,1] \times\left[4^{j}, 24^{j}\right] \subset Q_{j} \quad \text { and } \quad\left[\frac{1}{2} 2^{j}-1, \frac{1}{2} 2^{j}\right] \times\left[4^{j}, 24^{j}\right] \subset Q_{j} .
$$

We have for $j>2$ (for $j \leq 2$ we have $\left\langle f, \gamma_{j, l, k}\right\rangle=0$ and also $\left\langle f, \varphi_{k}^{c}\right\rangle=0$ )

$$
\begin{aligned}
\left\langle f, \gamma_{j, l, k}\right\rangle & =2^{-3 j / 2} \int_{\mathbb{R}^{2}} \hat{f}(\xi) \hat{\psi}_{j, l}^{c}(\xi) \exp \left(i R_{s_{j, l}}\left(4^{-j} k_{1}, 2^{-j} k_{2}\right)^{t} \cdot \xi\right) d \xi \\
& =\delta_{l 2^{j-2}} 2^{-3 j / 2} 2^{-j-j \varepsilon} \int_{[0,1] \times\left[4^{j}, 24^{j}\right]} \psi_{j, l}^{c}(\xi) \exp \left(i\left(4^{-j} k_{1} \xi_{2}+2^{-j} k_{2} \xi_{1}\right)\right) d \xi \\
& =\delta_{l 2^{j-2}} 2^{-3 j / 2} 2^{-j-j \varepsilon} \int_{[0,1] \times\left[4^{j}, 24^{j}\right]} \exp \left(i\left(4^{-j} k_{1} \xi_{2}+2^{-j} k_{2} \xi_{1}\right)\right) d \xi,
\end{aligned}
$$

where the last two equalities make use of (18) and (21). Similarly, we compute

$$
\begin{aligned}
\left\langle g, \gamma_{j, l, k}\right\rangle= & \delta_{l 2^{j-2}} 2^{-3 j / 2} 2^{-j-j \varepsilon} \int_{\left[\frac{1}{2} 2^{j}-1, \frac{1}{2} 2^{j}\right] \times\left[4^{j}, 24^{j}\right]} \exp \left(i\left(4^{-j} k_{1} \xi_{2}+2^{-j} k_{2} \xi_{1}\right)\right) d \xi \\
= & \delta_{l 2^{j-2}} 2^{-3 j / 2} 2^{-j-j \varepsilon} \int_{[0,1] \times\left[4^{j}, 24^{j}\right]} \\
& \quad \times \exp \left(i\left(4^{-j} k_{1} \xi_{2}+2^{-j} k_{2}\left(\xi_{1}+\frac{1}{2} 2^{j}-1\right)\right)\right) d \xi \\
= & \exp \left(i k_{2}\left(1 / 2-2^{-j}\right)\right)\left\langle f, \gamma_{j, l, k}\right\rangle .
\end{aligned}
$$

In particular this implies (iii), which is what we sought.

\section{Conclusion}

In this paper we studied stability properties of curvelet and ridgelet frames in mixedsmoothness Sobolev spaces. It turns out that curvelets are not suitable to characterize such spaces, while ridgelets are. It is straightforward to adapt our results to other 
systems based on parabolic scaling like for instance the shearlet transform. In future work we aim at constructing stable solvers for linear transport problems using ridgelet discretizations.

Acknowledgement The author thanks Christoph Schwab for useful discussions.

\section{References}

1. Borup, L., Nielsen, M.: Frame decompositions of decomposition spaces. J. Fourier Anal. Appl. 13, 39-70 (2007)

2. Candes, E.: Ridgelets: theory and applications. PhD thesis, Stanford University (1998)

3. Candes, E., Demanet, L.: The curvelet representation of wave propagators is optimally sparse. Commun. Pure Appl. Math. 58, 1472-1528 (2004)

4. Candes, E., Donoho, D.: Curvelets-a surprisingly effective nonadaptive representation for objects with edges. In: Curves and Surfaces. Vanderbilt University Press, Nashville (1999)

5. Candes, E., Donoho, D.: New tight frames of curvelets and optimal representations of objects with piecewise $C^{2}$ singularities. Commun. Pure Appl. Math. 57, 219-266 (2002)

6. Candes, E., Donoho, D.: Continuous curvelet transform: II. discretization and frames. Appl. Comput. Harmon. Anal. 19(2), 198-222 (2005)

7. Christensen, O.: An Introduction to Frames and Riesz Bases. Birkhäuser, Basel (2003)

8. Dahmen, W.: Wavelet and multiscale methods for operator equations. Acta Numer. 6, 55-228 (1997)

9. Do, M., Vetterli, M.: The contourlet transform: an efficient directional multiresolution image representation. IEEE Trans. Image Process. 14, 2091-2106 (2005)

10. Donoho, D.: Ridge functions and orthonormal ridgelets. J. Approx. Theory 111, 143-179 (1998)

11. Donoho, D.: Wedgelets: Nearly minimax estimation of edges. Ann. Stat. 27, 353-382 (1998)

12. Fadili, M., Starck, J.-L.: Curvelets and ridgelets. In: Encyclopedia of Complexity and Systems Science, pp. 1718-1738. Springer, Berlin (2009)

13. Feichtinger, H.-G., Gröbner, P.: Banach spaces of distributions defined by decomposition methods. I. Math. Nachr. 123, 97-120 (1985)

14. Guo, K., Kutyniok, G., Labate, D.: Sparse multidimensional representations using anisotropic dilation and shear operators. In: Wavelets and Splines, Athens, GA, 2005, pp. 189-201. Nasboro Press, Brentwood (2006).

15. Hörmander, L.: The Analysis of Linear Partial Differential Operators I. Springer, Berlin (2003)

16. Labate, D., Lim, W.-Q., Kutyniok, G., Weiss, G.: Sparse multidimensional representation using shearlets. In: Proceedings of the SPIE,3 pp. 254-262. SPIE Press, Bellingham (2005)

17. LePennec, E., Mallat, S.: Sparse geometric image representation with bandelets. IEEE Trans. Image Process. 14, 423-438 (2005)

18. Meyer, Y., Coifman, R.: Wavelets: Calderon-Zygmund and Multilinear Operators. Cambridge University Press, Cambridge (1997)

19. Modest, M.: Radiative Heat Transfer. Academic Press, San Diego (2003)

20. Smith, H.: A parametrix construction for wave equations with $C^{1,1}$-coefficients. Ann. Inst. Fourier 48, 797-835 (1998)

21. Widmer, G., Hiptmair, R., Schwab, C.: Sparse adaptive finite elements for radiative transfer. J. Comput. Phys. 227(12), 6071-6105 (2008) 